\title{
2 SARS-CoV-2 utilizes a multipronged strategy to suppress host
}

\section{3 protein synthesis}

4 Yaara Finkel $^{1,5}$, Avi Gluck ${ }^{1,5}$, Roni Winkler ${ }^{1,5}$, Aharon Nachshon ${ }^{1}$, Orel Mizrahi ${ }^{1}$, Yoav

5 Lubelsky $^{2}$, Binyamin Zuckerman ${ }^{2}$, Boris Slobodin $^{3}$, Yfat Yahalom-Ronen ${ }^{4}$, Hadas Tamir ${ }^{4}$, Igor

6 Ulitsky $^{2}$, Tomer Israely ${ }^{4}$, Nir Paran $^{4}$, Michal Schwartz $^{1 *}$ and Noam Stern-Ginossar ${ }^{{ }^{*}}$

$8 \quad{ }^{1}$ Department of Molecular Genetics, Weizmann Institute of Science, Rehovot 76100, Israel.

$9 \quad{ }^{2}$ Department of Biological Regulation, Weizmann Institute of Science, Rehovot 76100, Israel

$10{ }^{3}$ Department of Biomolecular Sciences, The Weizmann Institute of Science, Rehovot, Israel

$11{ }^{4}$ Department of Infectious Diseases, Israel Institute for Biological Research, Ness Ziona 74100,

12 Israel.

135 These authors contributed equally to this work

$14 *$ To whom correspondence should be addressed: michalsc@ weizmann.ac.il

15 or noam.stern-ginossar@weizmann.ac.il

16 


\section{Abstract:}

19 Severe acute respiratory syndrome coronavirus 2 (SARS-CoV-2) is the cause of the ongoing

20 coronavirus disease 19 (COVID-19) pandemic. Despite the urgent need, we still do not fully

21 understand the molecular basis of SARS-CoV-2 pathogenesis and its ability to antagonize innate

22 immune responses. Here, we use RNA-sequencing and ribosome profiling along SARS-CoV-2

23 infection and comprehensively define the mechanisms that are utilized by SARS-CoV-2 to

24 shutoff cellular protein synthesis. We show SARS-CoV-2 infection leads to a global reduction in

25 translation but that viral transcripts are not preferentially translated. Instead, we reveal that

26 infection leads to accelerated degradation of cytosolic cellular mRNAs which facilitates viral

27 takeover of the mRNA pool in infected cells. Moreover, we show that the translation of

28 transcripts whose expression is induced in response to infection, including innate immune genes,

29 is impaired, implying infection prevents newly transcribed cellular mRNAs from accessing the

30 ribosomes. Overall, our results uncover the multipronged strategy employed by SARS-CoV-2 to

31 commandeer the translation machinery and to suppress host defenses. 


\section{Introduction:}

34 Severe acute respiratory syndrome coronavirus 2 (SARS-CoV-2) is the cause of the ongoing coronavirus disease 19 (COVID-19) pandemic ${ }^{1,2}$. SARS-CoV-2 is an enveloped virus consisting

36 of a positive-sense, single-stranded RNA genome of $\sim 30 \mathrm{~kb}$. From the positive strand genomic

37 RNA, two overlapping open reading frames (ORFs) are translated, ORF1a (pp1a) and ORF1b

38 (pp1ab). The translation of ORF1b is mediated by a -1 frameshift that allows translation to

39 continue beyond the stop codon of ORF1a. These generate continuous polypeptides which are

40 cleaved into a total of 16 nonstructural proteins (NSP 1-16, Snijder et al., 2016; Sola et al., 2015;

41 Stadler et al., 2003; V'kovski et al., 2020). Among them are the subunits that constitute the

42 RNA-dependent RNA polymerase (RdRP), which in turn transcribes subgenomic RNAs that

43 contain a common 5' leader fused to different segments from the $3^{\prime}$ end of the viral genome ${ }^{3-5}$.

44 The different subgenomic RNAs encode 4 conserved structural proteins- spike protein (S),

45 envelope protein $(\mathrm{E})$, membrane protein $(\mathrm{M})$, nucleocapsid protein $(\mathrm{N})$ - and several accessory

46 proteins and non-canonical protein products ${ }^{7-9}$.

47 Translation of viral proteins relies solely on the cellular translation machinery; therefore, viruses

48 must commandeer this machinery to translate their own mRNAs. In addition, viruses need to

49 counteract the antiviral response cells mount upon infection ${ }^{10}$. The first line of defense applied

50 by infected cells engages the interferon (IFN) pathway, which amplifies signals resulting from

51 detection of intracellular viral components to induce a systemic anti-viral response. Specifically,

52 cells contain various sensors that detect the presence of viral RNAs and promote nuclear

53 translocation of transcription factors, leading to transcription and secretion of type I IFNs ${ }^{11}$.

54 Binding of IFN to its cognate receptor in autocrine and paracrine manners leads to the

55 propagation of the signal and to the transcription and translation of hundreds of interferon

56 stimulated genes that act to hamper viral replication at various stages of the viral life cycle ${ }^{12}$. In

57 the case of SARS-CoV-2, interferon response seems to play a critical role in pathogenesis ${ }^{13-17}$.

58 In addition, the extent to which SARS-CoV-2 suppresses the IFN response is a key characteristic

59 that distinguishes it from other respiratory viruses ${ }^{18,19}$.

60 Viruses utilize various strategies to cause shutoff of host mRNA translation ${ }^{10}$, including

61 hampering mRNA processing steps and export, inducing degradation of mRNAs and inhibiting 
62 translation. Coronaviruses (CoVs) are known to cause host shutoff ${ }^{10,20}$ and several strategies

63 have been proposed for how the beta CoVs may shut-off host protein synthesis and evade

64 immune detection. These include degradation of host mRNA in the nucleus or the cytosol and

65 inhibition of host translation ${ }^{21,22}$. Nonetheless, the extent to which SARS-CoV-2 uses these or

66 other strategies remains unclear.

67 NSP1 is the best characterized and most prominent coronavirus host shutoff factor ${ }^{23}$. Several

68 recent studies showed SARS-CoV-2 NSP1 binds the 40s ribosome and inhibits translation ${ }^{24-28}$.

69 In addition, other SARS-CoV-2 proteins were shown to interfere with cellular gene expression.

70 For example, one of the SARS-CoV-2 accessory proteins, ORF6, was suggested to disrupt

71 nucleocytoplasmic transport leading to inhibition of gene expression ${ }^{29}$ and several viral proteins

72 have been demonstrated to antagonize IFN-I production and signaling ${ }^{24,30}$. Although, different

73 SARS-CoV-2 proteins are implicated in host expression shutoff, a comprehensive picture of the

74 effect of SARS-CoV-2 infection on cellular gene expression is still lacking.

75 Here we employ RNA-sequencing and ribosome profiling along SARS-CoV-2 infection to

76 explore the mechanisms which the virus utilizes to interfere with host protein synthesis. We

77 reveal SARS-CoV-2 uses a multi-faceted approach to shutoff cellular protein production. SARS-

78 CoV-2 infection induces global translation inhibition but surprisingly the translation of viral

79 transcripts is not preferred over their cellular counterparts. Instead we reveal that infection leads

80 to accelerated cellular mRNAs degradation, likely conferred by NSP1. Viral transcripts are

81 refractory to these effects, an evasion potentiated by their 5'UTRs, enabling viral dominance

82 over the mRNA pool in infected cell. Finally, we show that the translation of transcripts whose

83 expression is induced in response to infection, including innate immune genes, is severely

84 impaired. Overall these findings reveal the key mechanisms SARS-CoV-2 is applying to

85 suppress cellular protein synthesis and host defenses. 


\section{Results:}

\section{Simultaneous monitoring of RNA levels and translation during SARS-CoV-2 infection}

To gain a detailed view of the changes that occur in viral and host translation over the course of SARS-CoV-2 infection, we infected Calu3 cells with SARS-CoV-2 at multiplicity of infection (MOI) of 3 and harvested infected cells at 3, 5, and 8 hours post infection (hpi) as well as uninfected cells. This high MOI infection results in infection of the majority of the cells and thus a synchronous cell population, allowing for molecular dissection of the events along infection. We designed our experiment to simultaneously monitor both RNA levels and translation (Figure 1A). Deep sequencing of mRNA (RNA-seq) generates a detailed depiction of transcript levels during infection and this was paired with ribosome profiling, which allows accurate measurement of protein synthesis by capturing the overall in-vivo distribution of ribosomes on a given message ${ }^{31,32}$. In order to assess the reproducibility of our experiments we prepared two independent biological replicates for the uninfected and 8hpi time points and both the mRNA and footprint measurements were reproducible (Figure S1A and S1B). Metagene analysis, in which gene profiles are aligned and then averaged, revealed the expected profiles of footprints along mRNAs; ribosome density accumulates from the start codon along the gene body ending at the first in-frame stop codon with pronounced accumulation of ribosomes at the initiation sites and 3 bp periodicity along the gene body (Figure S1C). Using this data, we quantitatively assessed the expression pattern of 8627 cellular transcripts and 12 canonical viral ORFs that are expressed from the genomic and sub-genomic RNAs along SARS-CoV-2 infection. SARS-CoV-2's unprecedented dominance over the mRNA pool. At 8 hpi viral mRNAs comprise almost $90 \%$ of the mRNAs in infected cells (Figure 1B). Surprisingly, however, at the same time

110 point, viral mRNAs account for only $\sim 34 \%$ of the RNA fragments engaged with ribosomes in the

111 cells (Figure 1C). In order to quantitatively evaluate the ability of SARS-CoV-2 to co-opt the

112 host ribosomes we calculated the relative translation efficiency (TE) of viral and cellular RNA

113 along infection. TE is defined as the ratio of footprints to mRNA reads for a given gene and

114 reflects how well a gene is being translated. We then compared the TE of human genes to that of 115 viral genes at each of the time points along infection. The analysis shows that at 3 hpi viral gene 
116 translation efficiencies fall within the general range of cellular gene translation. This indicates

117 that when infection initiates, viral transcripts are translated with efficiencies similar to those of

118 host transcripts. As infection progresses, viral gene translation efficiency relative to cellular

119 genes is significantly reduced (Figure 1D). Since it seems improbable that the virus will promote

120 conditions that are unfavorable to the translation of its mRNA, this relative reduction in

121 translation of viral genes at 5 and 8 hpi may indicate that not all viral RNAs are accessible for

122 translation. Since double-membrane sealed replication compartments are formed to

123 accommodate viral genome replication ${ }^{33}$, an appealing possibility is that these compartments

124 sequester a sizable fraction of the viral RNA molecules and thus prevent them from being a part

125 of the translated mRNA pool.

126 By their nature, deep sequencing measurements provide relative values but not absolute

127 quantification of RNA and translation levels. Since SARS-CoV-2 encoded protein, NSP1, was

128 recently shown to interfere with translation by blocking the mRNA entry channel of ribosomes

$129^{25-28}$, and since the extent to which SARS-CoV-2 interferes with the overall levels of cellular

130 mRNA was not assessed, we next examined if SARS-CoV-2 infection affects global translation

131 and RNA levels. To quantify absolute translation levels, we measured nascent protein synthesis

132 levels using an analogue of puromycin, O-Propargyl Puromycin (OPP), which incorporates into

133 elongating polypeptide chains ${ }^{34}$. The nascent polypeptides with incorporated OPP are

134 fluorescently-labeled via a Click reaction and the levels of OPP incorporation into elongating

135 polypeptides is quantified by flow cytometry (Figure S2A and S2B). We infected Calu3 cells

136 with SARS-CoV-2 at an MOI of 3 and measured nascent protein synthesis levels in uninfected

137 cells and at 3,5 and 8 hpi. We observed a significant reduction in global translation levels

138 already at 3 hpi which was augmented with time, and at 8 hpi translation activity was reduced by

$13950 \%$ (Figure 1E). In parallel we measured the total RNA levels and rRNA levels extracted from

140 uninfected cells and at 3, 5, and $8 \mathrm{hpi}$. This analysis illustrated there are no major changes in

141 total RNA or in rRNA levels along SARS-CoV-2 infection (Figure S3A and S3B). Since the vast

142 majority of RNA in cells originates from rRNA and this dominance of rRNA may mask changes

143 in mRNA levels we sequenced total RNA, without rRNA depletion, to assess the relative

144 abundance of cellular and viral mRNAs in uninfected cells and at 3, 5, and 8 hpi. This analysis

145 demonstrates that the pool of mRNA molecules relative to rRNA is growing during infection, 
146 due to the massive production of viral transcripts but at the same time the relative fraction of

147 cellular mRNA is reduced by approximately 2-fold (Figure1F). This suggests that during

148 infection there is both massive production of viral transcripts and a concomitant substantial

149 reduction in the levels of cellular transcripts.

150 Next, we quantitatively assessed the expression pattern of cellular genes along SARS-CoV-2

151 infection. We clustered the mRNA levels of genes that showed the most significant changes

152 along infection using partitioning clustering, allowing grouping of cellular transcripts into three

153 distinct classes based on similarities in temporal expression profiles in the RNA-seq. Overall, we

154 found that changes in ribosome footprints tracked the changes in transcript abundance (Figure

$1551 \mathrm{G})$, with some exceptions that will be discussed in detail below. This shows that part of the

156 reduction in cellular protein synthesis is driven by the reduction in cellular RNA levels.

157 Interestingly, although the levels of the majority of host transcripts were reduced during SARS-

158 CoV-2 infection, we identified numerous transcripts that were significantly elevated (Figure 1G,

159 cluster 3). We carried out pathway enrichment analysis for each of these three clusters. As

160 expected, the group of upregulated mRNAs (cluster 3) was significantly enriched with genes

161 related to immune response, including Toll receptor Signaling, chemokine and cytokine

162 signaling (Figure 1H and Table S1) and these upregulated genes include IL6 and IL8 which play

163 a significant role in the pathogenesis of SARS-CoV-2 ${ }^{35}$ and several IFN stimulated genes like,

164 IFIT1, 2 and 3, IRF1, ISG15 and TNF alpha induced proteins. These measurements and

165 analyses reveal that the shutoff in host protein synthesis is driven by several mechanisms

166 including; general reduction in the translation capacity of infected cells and reduction in the

167 levels of most cellular mRNAs.

169 Viral gene expression dynamics along infection

170 We next analyzed viral gene expression dynamics along SARS-CoV-2 infection. Viral ORFs are

171 translated from the $30 \mathrm{~kb}$ genomic RNA or from a nested series of subgenomic RNAs that

172 contain a common $5^{\prime}$ leader fused to different segments from the $3^{\prime}$ end of the viral genome

173 (Figure 2A and V'kovski et al., 2020). Viral transcripts and translation levels significantly 
174 increased from 3 to 5 hpi but the relative abundance of some viral transcripts and their translation

175 rates were reduced from 5 to $8 \mathrm{hpi}$ (Figure $2 \mathrm{~B}$ and $2 \mathrm{C}$ ). This relative reduction in translation rates

176 of some viral transcripts prompted us to assess the relative translation efficiency of viral genes

177 along infection. Since, as indicated above, the translation efficiency of viral genes compared to

178 their cellular counterparts is relatively reduced along infection (Figure 1D), here we examined

179 how the translation of viral genes is distributed between different viral transcripts at different

180 times post infection. This focused analysis revealed an interesting connection between changes

181 in translation efficiency of viral ORFs and their relative location on the viral genome. ORFs that

182 are located at the 5 ' end of the genome showed relative increase in their translation efficiency

183 along infection whereas ORFs that are encoded towards the 3' end of the genome showed

184 relative reduction in their translation efficiencies and ORFs located in the middle of genome

185 showed no major changes in their relative translation efficiency (Figure 2D). Since all viral

186 subgenomic RNAs share the same 5'UTR these differences in translation capacity according to

187 the location in the genome point to a potential unappreciated role for the 3'UTR (the 3' portion

188 of which is shared between all transcripts) or for the length of viral transcripts, which varies

189 greatly between viral transcripts (Figure 2A). To examine if this phenomenon reflects a general

190 beta CoVs feature, we analyzed the expression and translation of Mouse Hepatitis Virus strain

191 A59 (MHV) ORFs along MHV infection using published RNA-seq and ribosome profiling data

192 along MHV infection ${ }^{36}$. MHV has a similar genomic organization (Figure 2E) and the increase

193 in viral mRNA and translation between 5 and 8 hpi varied between viral ORFs (Figure $2 \mathrm{~F}$ and

194 2G). Although this phenomenon was not as strong, also in the case of MHV, ORFs that are

195 located at the $5^{\prime}$ end of the genome showed relative increase or no change in their translation

196 efficiency along infection whereas ORFs that are encoded towards the 3' end of the genome

197 showed relative reduction in their translation efficiency (Figure 2H). Together these results

198 indicate that the 3'UTR or viral gene length are likely regulating the translation efficacy of viral

199 mRNAs along infection.

200 Most SARS-CoV-2 ORFs are 3'-proximal and translated from dedicated subgenomic mRNA

201 (Figure 2A). However several subgenomic RNAs encode for additional out-of-frame ORFs,

202 likely via a leaky scanning mechanism ${ }^{9,37-39}$. These include ORFs $7 b$ and ORF9b, which are

203 translated from the ORF7a and N subgenomic RNAs, and two ORFs we recently identified by 
204 ribosome profiling ${ }^{9}$, that are supported by additional evidence ${ }^{37,39}$, ORF3c and ORFS.iORF

205 that are translated from ORF3a and ORF-S subgenomic RNAs. Since scanning efficiency can be

206 regulated by stress conditions ${ }^{40}$ we examined if the ratio between the translation of a $3^{\prime}$-proximal

207 ORF and its corresponding out-of-frame ORF (encoded by the same subgenomic RNA) changes

208 during infection. Since ORF9b expression was low in our measurements it was excluded from

209 this analysis. The translation of ORF7b, ORF3c and ORFS.iORF correlated with the expression

210 of the 3'-proximal main ORF, indicating there are no major changes in the efficiency of ribosome

211 scanning of viral transcripts along infection (Figure 2I).

213 Cellular mRNAs are degraded during SARS-CoV-2 infection

214 Our results indicate that the levels of the majority of cellular RNA are reduced during SARS-

$215 \mathrm{CoV}-2$ infection and this reduction contributes to the shutoff of cellular protein synthesis.

216 Reduction in cellular RNA levels could be due to interference with RNA production or

217 accelerated RNA degradation. To explore the molecular mechanism, we analyzed if the

218 reduction in cellular transcripts is associated with their subcellular localization. We used

219 measurements of the subcellular localization of transcripts by cytoplasmic and nuclear

220 fractionation $^{41}$ to assess the importance of subcellular localization. The levels of transcripts that

221 mostly localize to the cytoplasm were more reduced in infected cells compared to transcripts that

222 are mostly nuclear (Figure 3A) and there was a clear correlation between subcellular localization

223 and the extent of reduction in transcript levels following SARS-CoV-2 infection (Figure S4A).

224 Furthermore, compared to transcripts encoded in the nuclear genome, mitochondrial encoded

225 transcripts were refractory to the effects of SARS-CoV-2 infection (Figure 3B). The specific

226 sensitivity of cytosolic transcripts implies these transcripts may be specifically targeted during

227 SARS-CoV-2 infection. In CoVs, the most prominent and well characterized cellular shutoff

228 protein is NSP1 ${ }^{23}$. So far, studies on SARS-CoV-2 NSP1 demonstrated it restricts translation by

229 directly binding to the ribosome 40 S subunit $^{25-28}$, thereby globally inhibiting translation

230 initiation. For SARS-CoV, on top of this translation effect, NSP1 interactions with the 40S was

231 also shown to induce cleavage of translated cellular mRNAs, thereby accelerating their turnover

232 . To examine if the reduction in cellular transcripts in SARS-CoV-2 infected cells is coupled to 
233 their translation we compiled a list of 14 long non-coding RNAs (lncRNAs) that localize to the

234 cytoplasm and are well expressed in our data but, as expected, poorly translated (Figure S4B).

235 Relatively to cellular mRNAs, cytoplasmic lncRNAs were less affected by SARS-CoV-2

236 infection (Figure 3C), indicating accelerated turnover of cellular transcripts in infected cells may

237 be related to their translation. Recently, ribosome profiling and RNA-seq were conducted on

238 cells transfected with NSP1 ${ }^{42}$. Analysis of the RNA expression from this data revealed that

239 ectopic NSP1 expression leads to weaker but similar signatures to the ones we identified in

240 infected cells; stronger reduction of cytosolic transcripts compared to nuclear transcripts,

241 stronger sensitivity of nuclear encoded transcripts, and stronger reduction of translated mRNA

242 compared to cytosolic lncRNAs (Figure S5A-C).

243 We further noticed SARS-CoV-2 infection leads to increased levels of intronic reads in many

244 cellular transcripts (Figures 3D and 3E) indicating SARS-CoV-2 may interfere with cellular

245 mRNA splicing, as was recently suggested ${ }^{24}$. However, massive degradation of mature cytosolic

246 mRNAs may also generate a relative increase in intronic reads. We therefore analyzed the ratio

247 of intronic and exonic reads relative to rRNA. Whereas relative to rRNA levels, exonic reads

248 showed drastic reduction along SARS-CoV-2 infection, the intronic reads levels showed a more

249 subtle change (Figure 3F). Furthermore, the increase in the ratio of intronic to exonic reads was

250 greater in genes whose expression was reduced along infection compared to genes whose

251 expression was induced (Figure 3G and Figure S6A), illustrating the relative increase in intronic

252 reads is mostly independent of newly transcribed RNAs. Finally, we also detected more intronic

253 reads in cells that exogenously expressed NSP1 ${ }^{42}$ (Figure S6B). Together these results indicate

254 that the increase in intronic reads compared to exonic reads during SARS-CoV-2 infection is

255 largely driven by accelerated degradation of mature cellular transcripts that leads to relative

256 reduction in exonic reads. It is likely that SARS-CoV-2 also directly regulates splicing

257 efficiency, as was recently proposed ${ }^{24}$, but this effect seems more subtle. Together these

258 findings indicate SARS-CoV-2 infection leads to accelerated degradation of cytosolic cellular

259 mRNAs and that this degradation significantly affects the production of cellular proteins.

\section{SARS-CoV-2 5'UTR protects viral RNA from NSP1 induced RNA degradation}


261 An important aspect of host shutoff during infection is the ability of the virus to hamper the

262 translation of cellular transcripts while recruiting the ribosome to its own transcripts. Although it

263 has been suggested that SARS-CoV-2 mRNAs are refractory to the translation inhibition induced

264 by NSP1 ${ }^{24,43}$ our measurements indicate that SARS-CoV-2 transcripts are not preferentially

265 translated in infected cells (Figure 1D and ${ }^{9}$ ), that RNA degradation plays a prominent role in

266 remodeling the mRNA pool in infected cells and that SARS-CoV-2 dominates the mRNA pool.

267 All SARS-CoV-2-encoded subgenomic RNAs contain a common 5' leader sequence that is

268 added during negative-strand synthesis ${ }^{44}$. We therefore explored whether the 5'UTR sequence

269 protects viral mRNAs from NSP1 induced degradation. We fused the viral 5'UTR sequence or a

270 short control 5'UTR to the 5' end of a GFP reporter (Figure 4A) and transfected these constructs

271 together with expression vectors encoding NSP1 or NSP2 (that was used as a control) into 293T

272 cells. We found that NSP1 expression suppresses the production of the control-GFP but not of

273 the 5'UTR-containing GFP (Figure 4B and 4C). We extracted RNA from these cells and

274 observed that the NSP1 induced reduction in control-GFP expression was associated with 15-

275 fold reduction in the RNA GFP levels whereas the levels of the GFP RNA with the SARS-CoV-

2762 5'UTR were not reduced, and were even slightly increased, by NSP1 expression (Figure 4D).

277 The plasmid we used also contains an mCherry reporter expressed from an independent

278 promoter. Reassuringly NSP1 also induces a reduction in both mCherry protein (Figure S7A and

279 S7B) and RNA levels (Figure S7C) when compared to NSP2. These results indicate that the 5'

280 UTR of viral RNAs provides them protection from NSP1 induced degradation and that this

281 protection contributes to the ability of the virus to dominate the mRNA pool in infected cells.

\section{The translation efficiency of transcriptionally induced genes is impaired during infection}

283 Our results so far exemplify how SARS-CoV-2 remodels the transcript pool in infected cells. To

284 quantitatively evaluate the role of translational control along SARS-CoV-2 infection, we

285 calculated relative translation efficiency (TE, ratio of footprints to mRNAs for a given gene)

286 along infection. We then centered on genes that showed the strongest change in their relative TE

287 along infection. We clustered these genes into four clusters, based on similarity in their temporal

288 TE profiles, which largely reflect genes whose relative TE is reduced along infection and genes

289 whose relative TE is increased. The mRNA and footprint temporal profiles of these genes reveal

290 a clear signature; the genes whose relative TE along infection was reduced were genes whose 
mRNA increased during infection but did not show a corresponding increase in footprints

292 (Figure 5A and Figure S8). These genes were enriched in immune response genes (FDR $<10^{-4}$ )

293 like IRF1, IL-6 and CXCL3. Comparing changes in mRNA and TE levels of cellular genes along

294 infection demonstrates that generally transcripts which are transcriptionally induced following

295 infection show a reduction in their relative TE and vice versa (Figure 5B). This inability of RNA

296 that is elevated in response to infection to reach the ribosomes, may explain why infected cells

297 fail to launch a robust IFN response ${ }^{13,14}$. These data demonstrate that the overall capacity of

298 infected cells to generate new proteins is severely impaired.

\section{Discussion:}

Many viruses developed varied and sophisticated mechanisms to repress host mRNA translation in order to block the cellular innate immune response and concomitantly allow the translation of viral mRNAs. Specifically, CoVs have evolved specialized mechanisms to hijack the host gene expression machineries, including inhibition of host protein synthesis and induction of endonucleolytic cleavage of host mRNAs ${ }^{23,45}$. In the case of SARS-CoV-2 several viral ORFs have been suggested to interfere with viral gene expression ${ }^{24-28,30}$, but what occurs during viral infection remained an open question.

Using unbiased measurements of translation and RNA expression along SARS-CoV-2 infection, we identified three major courses by which SARS-CoV-2 interferes with cellular gene

310 expression in infected cells; 1. global inhibition of protein translation, 2. degradation of cytosolic

311 cellular transcripts and 3. specific translation inhibition of newly transcribed mRNAs.

312 Disruption of cellular protein production using these three components may represent a multi-

313 pronged mechanism that synergistically acts to suppress the host antiviral response (Figure 5C).

314 These mechanisms may explain the molecular basis of the potent suppression of IFN response 315 observed in animal models and in severe COVID-19 patients ${ }^{15,18}$.

316 Despite differences in protein size and mode of action, NSP1 proteins from alpha- and beta317 CoVs suppress host gene expression ${ }^{21,22,46}$, making Nsp1 a central host shutoff factor of CoVs.

318 Indeed, several studies focused on SARS-CoV-2 encoded NSP1, showing it binds to the 40S 
ribosomal subunit, leading to reduction in mRNA translation both in vitro and in cells ${ }^{24-28}$. Still,

320 how the virus can overcome the NSP1-mediated block of translation and how NSP1 effects come

321 into play during infection remained unclear. We reveal here that in resemblance to what had been

322 described for SARS-CoV NSP1, SARS-CoV-2 NSP1 induces shutdown of host protein

323 translation by two mechanisms: first, it stalls canonical mRNA translation as was reported by

324 others ${ }^{24-28,41}$ leading to general reduction in the translation capacity of infected cells. Second,

325 NSP1 leads to accelerated cellular mRNA degradation. SARS-CoV NSP1 induces

326 endonucleolytic cleavage and subsequent degradation of host mRNAs and this activity depends

327 on its binding to the $40 \mathrm{~S}$ ribosome subunit ${ }^{21,47}$. Our results indicate a similar mechanism

328 operates in SARS-CoV-2 infected cells as we show cytosolic RNAs, specifically ones that are

329 being translated, are more susceptible to SARS-CoV-2-mediated degradation. several studies

330 have shown that mRNAs with viral 5' UTR are translated more efficiently compared to control

331 5'UTR in the presence of NSP1 ${ }^{24,27,4326}$ but it was further demonstrated that NSP1 inhibits

332 translation of both cellular and a viral 5' UTR-containing reporter mRNA 2726 implying viral

333 mRNAs do not simply evade translation inhibition in the context of the 5' UTR sequence. Our

334 measurements from infected cells support these findings as we show viral RNA are not

335 preferentially translated in infected cells. Instead, using a reporter, we show that the viral 5'UTR

336 renders mRNAs refractory to RNA degradation mediated by NSP1. Together these results

337 support a model in which NSP1 acts as a strong inhibitor of translation, tightly binding

338 ribosomes and reducing the pool of available ribosomes that can engage in translation. At the

339 same time ribosome bound NSP1 leads to accelerated degradation of cellular but not of viral

340 mRNAs providing means for viral mRNA to quickly take over and dominate the mRNA pool.

341 This accumulation of SARS-CoV-2 mRNAs explains how infected cells switch their translation

342 towards viral mRNAs. In support of this model, we show that within 8 hours $88 \%$ of the mRNAs

343 in infected cells is viral. Notably, interactions between SARS-CoV NSP1 and the viral 5' UTR

344 prevent NSP1-induced RNA cleavage ${ }^{47}$, indicating that likely similar mechanisms are applied

345 by SARS-CoV and SARS-CoV-2 viruses.

346 On top of the general translation reduction and cellular mRNA degradation which are likely

347 mediated by NSP1, we also observed that in mRNAs that are being induced in response to

348 infection there is no corresponding increase in footprints, indicating these newly generated 
transcripts are less likely to engage with ribosomes. Since mRNAs that are induced in infection

350 are enriched in cytokines and IFN induced genes, this distinctive mechanism may provide

351 additional explanation why infected cells fail to mount an efficient anti-viral response. Although

352 we do not know the underlying molecular mechanism, one appealing hypothesis is viral

353 inhibition of cellular mRNA nuclear export. Since SARS-CoV-2 replicates in the cytoplasm,

354 inhibiting the nuclear export of mRNAs can provide unique advantages as it will specifically

355 inhibit cellular mRNA translation and more explicitly it will lead to suppression of the host's

356 antiviral response genes which are transcriptionally induced and therefore fully depend on

357 efficient nuclear export. ORF6 was recently shown to co-purify with host mRNA export factors

$358 \quad 48$ and by over expression it disrupts nucleocytoplasmic export ${ }^{29}$, providing a candidate that may

359 explain the phenotype we observe in infection. Another possibility is that NSP16, which was

360 recently shown to inhibit cellular RNA splicing ${ }^{24}$, is driving this phenotype as interference with

361 splicing will prevent efficient nuclear export. Indeed, we show here that infection leads to

362 increased levels of intronic reads in many cellular transcripts. Although our analysis reveals

363 some of this signature is attributed to accelerated degradation of mature cellular transcripts it is

364 likely there is also a processing and splicing defect that leads to more intronic reads and

365 eventually aberrant export.

366 Overall, our study provides an in-depth picture of how SARS-CoV-2 efficiently interferes with

367 cellular gene expression, leading to shutdown of host protein production using a multipronged

368 strategy. 


\section{Figure legends:}

371 Figure 1: Global reduction of translation and in cellular mRNA levels along SARS-CoV-2

372 Infection

373 (A) Calu3 cells were left uninfected or were infected with SARS-CoV-2 (MOI=3) for 3, 5 or 8 374 hours and harvested for RNA-seq and for Ribo-seq. (B and C) Percentage of reads that aligned 375 to human or viral transcripts from the sum of aligned reads shown for mRNAs (B) and footprints

376 (C) in uninfected cells and in cells harvested at 3, 5 and 8 hpi. (D) Cumulative TE distribution 377 among well-expressed human (black points) and viral (colored points) genes at 3, 5 and 8 hpi.

378 (E) Protein synthesis measurement by flow cytometry of Calu3 cells infected with SARS-CoV-2 $379(\mathrm{MOI}=3)$ for 3, 5 and $8 \mathrm{hpi}$ or an uninfected control following O-Propargyl Puromycin (OPP) 380 incorporation and fluorescent labelling. (F) Percent of reads that aligned to the human or viral 381 transcripts from the sum of total RNA reads in uninfected cells and in cells harvested at, 3, 5 and 3828 hpi. (G) Heat map presenting relative mRNA and footprints expression of well-expressed 383 human transcripts that showed the most significant changes in their mRNA levels at 8 hpi 384 relative to uninfected, across time points during SARS-CoV-2 infection. Shown are expression 385 levels scaled by gene after partitioning clustering. Three main clusters are marked on the right. 386 (H) Summary of pathway enrichment analysis of genes enriched in cluster 3 (upregulated genes). 387 Dot size reflects the number of genes from each pathway included in the tested set, and dot color 388 reflects the false discovery rate (FDR) of the pathway enrichment.

Figure 2: Changes in viral gene expression along SARS-CoV-2 and MHV infection

391 (A) Schematic presentation of the SARS-CoV-2 genome organization, the subgenomic mRNAs 392 and the main ORFs. (B and C) RNA (B) and translation levels (C) of each of the SARS-CoV-2 393 canonical ORFs at 3, 5 and 8 hpi. (D) Relative translation efficiency of each canonical viral ORF 394 along infection. Genes are divided to three groups based on their physical location along the 395 genome. (E) Schematic presentation of the MHV subgenomic mRNAs, and the main ORFs. 396 ORFs that are not expressed in the strain that was used for ribosome profiling (MHV-A59) are 
marked by empty rectangles. (F and G) RNA (F) and translation levels (G) of each of the MHV

398 ORFs at 2.5, 5 and 8 hpi. Expression was calculated from ${ }^{36}$. (H) Relative translation efficiency

399 of each canonical viral ORF along infection. Genes are divided to two groups based on their

400 location on the genome. (I) Relative translation efficiency of the main ORFs (ORF7a, S and

401 ORF3a, labeled by circle) and the out of frame ORFs (ORF7b, S.iORF and ORF3c, labeled by

402 diamond) of the S, ORF3a and ORF7a subgenomic transcripts, respectively, along SARS-CoV-

4032 infection. Translation levels were calculated from ribosome densities using ORF-RATER ${ }^{49}$.

\section{Figure 3: Cytosolic cellular RNAs are degraded during SARS-CoV-2 infection}

(A) RNA level fold change of cellular RNAs at different time points after infection relative to

uninfected cells. RNAs were grouped to ten bins based on their cytosol to nucleus localization

408 ratio $^{41}$. (B) The change in RNA levels of nuclear encoded or mitochondrial encoded RNAs at

409 different time points after infection relative to uninfected cells. (C) The change in RNA

410 expression of cytosolic lncRNAs and protein coding mRNAs at different time points after

411 infection relative to uninfected cells. (D) RNA reads on exons and introns of the end of IL-32

412 gene from uninfected cells and at 8 hpi. (E) Box plots presenting the ratio of intronic to exonic

413 reads for each gene in uninfected cells and at the different time points along SARS-CoV-2

414 infection. (F) The \% of reads that align to exonic or intronic regions relative to rRNA abundance

415 along SARS-CoV-2 infection. (H) Histograms showing the change in the ratio of intronic to

416 exonic reads of cellular genes at 8hpi relative to uninfected cells. Genes are divided according to

417 the three clusters shown in figure $1 \mathrm{G}$ (representing different expression pattern along infection).

419 Figure 4: The viral 5' UTR protects mRNA from NSP1-mediated degradation

420 (A) $293 \mathrm{~T}$ cells were transfected with expression vectors encoding either NSP1 or NSP2 (as a 421 control) and with a GFP reporter (GFP (-) 5'UTR) or a GFP reporter that includes the viral 422 5'UTR (GFP (+) 5'UTR). (B) Microscopy images of cells co-transfected with NSP2 (top) or 423 NSP1 (bottom) together with either GFP (-) 5'UTR or GFP (+) 5'UTR. Scale bars are 100 $\mu \mathrm{m}$. 
424 (C) Flow cytometry analysis of cells co-transfected with NSP1 or NSP2 together with either GFP

425 (-) 5'UTR or GFP (+) 5'UTR. (D) Relative GFP RNA levels from GFP (-) 5'UTR or GFP (+)

426 5'UTR in cells expressing NSP1 or NSP2 as measured by quantitative RT-PCR. Data points

427 show measurement of biological replicates. Shown is one representative experiment out of two

428 performed.

430 Figure 5: The translation of induced transcripts is impaired during infection

431 (A) Heat map presenting relative TE, mRNA and footprints (FP) of human genes that showed the 432 most significant changes in their relative TE along SARS-CoV-2 infection. Shown are relative 433 expression ratios after partitioning clustering based on changes in relative TE values. (B) Scatter 434 plot presenting cellular transcript levels in uninfected cells compared to 8hpi. Genes are colored 435 based on the relative change in their TE between uninfected and 8hpi. Central cytokines and IFN 436 stimulated genes are labeled. (C) A model of how SARS-CoV-2 suppresses host gene expression 437 through multi-pronged approach: 1. Global translation reduction; 2. Degradation of cytosolic 438 cellular mRNAs; 3. Specific translation inhibition of newly synthesized cellular mRNAs.

\section{Acknowledgements}

442 We thank Stern-Ginossar lab members for providing valuable feedback. We thank Nevan

443 Krogan for the SARS-CoV-2 ORFs expression plasmids, Ghil Jona and Weizmann Bacteriology 444 and Genomic Repository Units for technical assistance, Shay Weiss for support and guidance in 445 assays involving SARS-CoV-2. This study was supported by Miel de Botton. Work in the Stern-

446 Ginossar lab is supported by a European Research Council starting grant (StG-2014-638142).

447 N.S-G is an incumbent of the Skirball Career Development Chair in New Scientists and is a 448 member of the European Molecular Biology Organization (EMBO) Young Investigator Program.

449 The authors declare no competing interests. 


\section{Methods}

\section{$451 \quad$ Cells and viruses}

452 Calu3 cells (ATCC HTB-55) were cultured in 6-well or 10cm plates with RPMI supplemented 453 with 10\% fetal bovine serum (FBS), MEM non-essential amino acids, 2mM L-Glutamine,

454 100Units/ml Penicillin and 1\% Na-pyruvate. Monolayers were washed once with RPMI without 455 FBS and infected with SARS-CoV-2 virus, at a multiplicity of infection (MOI) of 3, in the 456 presence of $20 \mu \mathrm{g}$ per ml TPCK trypsin (Thermo scientific). Plates were incubated for 1 hour at $45737^{\circ} \mathrm{C}$ to allow viral adsorption. Then RPMI medium supplemented with $2 \%$ fetal bovine serum, 458 MEM non-essential amino acids, L glutamine and penicillin-streptomycin-Nystatin at $37^{\circ} \mathrm{C}, 5 \%$ 459 CO2 was added to each well. SARS-CoV-2 BavPat1/2020 Ref-SKU: 026V-03883 was kindly 460 provided by C. Drosten, Charité-Universitätsmedizin Berlin, Germany. It was propagated (5 461 passages) and tittered on Vero E6 cells and then sequenced ${ }^{9}$ before it was used. Handling and 462 working with SARS-CoV-2 virus was conducted in a BSL3 facility in accordance with the 463 biosafety guidelines of the Israel Institute for Biological Research. The Institutional Biosafety 464 Committee of Weizmann Institute approved the protocol used in these studies.

467 For RNA-seq, cells were washed with PBS and then harvested with Tri-Reagent (Sigma468 Aldrich), total RNA was extracted, and poly-A selection was performed using Dynabeads 469 mRNA DIRECT Purification Kit (Invitrogen) mRNA sample was subjected to DNAseI 470 treatment and 3' dephosphorylation using FastAP Thermosensitive Alkaline Phosphatase 471 (Thermo Scientific) and T4 PNK (NEB) followed by 3' adaptor ligation using T4 ligase (NEB). 472 The ligated products used for reverse transcription with SSIII (Invitrogen) for first strand cDNA 473 synthesis. The cDNA products were 3' ligated with a second adaptor using T4 ligase and 474 amplified for 8 cycles in a PCR for final library products of 200-300bp. For Ribo-seq libraries, 475 cells were treated with $100 \mu \mathrm{g} / \mathrm{mL} \mathrm{CHX}$ for 1 minute. Cells were then placed on ice, washed 476 twice with PBS containing $100 \mu \mathrm{g} / \mathrm{mL}$ CHX, scraped from $10 \mathrm{~cm}$ plates, pelleted and lysed with 
477 lysis buffer ( $1 \%$ triton in $20 \mathrm{mM}$ Tris $7.5,150 \mathrm{mM} \mathrm{NaCl}, 5 \mathrm{mM} \mathrm{MgCl} 2,1 \mathrm{mM}$ dithiothreitol 478 supplemented with $10 \mathrm{U} / \mathrm{ml}$ Turbo DNase and $100 \mu \mathrm{g} / \mathrm{ml}$ cycloheximide). After lysis samples

479 stood on ice for $2 \mathrm{~h}$ and subsequent Ribo-seq library generation was performed as previously

480 described ${ }^{50}$. Briefly, cell lysate was treated with RNAseI for $45 \mathrm{~min}$ at room temperature

481 followed by SUPERase-In quenching. Sample was loaded on sucrose solution (34\% sucrose,

$48220 \mathrm{mM}$ Tris $7.5,150 \mathrm{mM} \mathrm{NaCl}, 5 \mathrm{mM} \mathrm{MgCl} 2,1 \mathrm{mM}$ dithiothreitol and $100 \mu \mathrm{g} / \mathrm{ml}$ cycloheximide)

483 and spun for $1 \mathrm{~h}$ at 100K RPM using TLA-110 rotor (Beckman) at 4c. Pellet was harvested using

484 TRI reagent and the RNA was collected using chloroform phase separation. For size selection,

$48515 \mathrm{uG}$ of total RNA was loaded into $15 \%$ TBE-UREA gel for 65min, and 28-34 footprints were

486 excised using 28 and 34 flanking RNA oligos, followed by RNA extraction and ribo-seq

487 protocol $^{50}$

489 Sequence alignment, metagene analysis

490 Sequencing reads were aligned as previously described ${ }^{51}$. Briefly, linker

491 (CTGTAGGCACCATCAAT) and poly-A sequences were removed and the remaining reads

492 were aligned to the Hg19 and to the SARS-Cov-2 genome (Genebank NC_045512.2) with 3

493 changes to match the used strain (BetaCoV/Germany/BavPat1/2020 EPI_ISL_406862),

494 241:C->T, 3037:C->T, 23403:A->G]. Alignment was performed using Bowtie v1.1.2 ${ }^{52}$ with

495 maximum two mismatches per read. Reads that were not aligned to the genome were aligned to

496 the transcriptome and to SARS-CoV-2 junctions that were recently annotated ${ }^{44}$. The aligned

497 position on the genome was determined as the 5' position of RNA-seq reads, and for Ribo-seq

498 reads the p-site of the ribosome was calculated according to read length using the off-set from

499 the 5' end of the reads that was calculated from canonical cellular ORFs. The offsets used are

$500+12$ for reads that were $28-29 \mathrm{bp}$ and +13 for reads that were $30-33 \mathrm{bp}$. Reads that were in

501 different length were discarded. In all figures presenting ribosome densities data, all footprint

502 lengths (28-33bp) are presented. 
503 Junctions spanning reads were quantified using STAR 2.5.3a aligner ${ }^{53}$, with running flags as

504 suggested by ${ }^{44}$, to overcome filtering of non-canonical junctions. Reads aligned to multiple

505 locations were discarded ${ }^{44}$.

506 For the metagene analysis only genes with more than 10 reads were used. For each gene,

507 normalization was done to its maximum signal and each position was normalized to the number

508 of genes contributing to the position.

$509 \quad$ Filtering of genes, quantification and RPKM normalization

510 For analysis of cellular genes, the genes were filtered according to the number of reads as

511 follows. The number of reads on a gene in each replicate, from at least one of the conditions

512 (uninfected or $8 \mathrm{hr}$ ) had to be greater than 50 for the mRNA and greater than 25 for the FP. For

513 heatmaps and clustering, gene filtering was done according to mRNA as described above, and

514 genes with zero reads in any of the samples (mRNA and FP of all time points) were excluded.

515 Histone genes were excluded. Read coverage on genes was normalized to units of RPKM in

516 order to normalize for gene length and for sequencing depth. For analysis comparing host and

517 viral genes, the RPKM was calculated based on the total number of reads of both the host and the

518 virus. For analysis concentrating on cellular genes alone, the RPKM was calculated based on the

519 total number of host reads, and the RPKM values were further scaled to keep the number of total

520 reads equal across samples. The scale factors for RNA-seq were calculated from the ratio of the

521 host mRNA reads to total reads, including viral, rRNA and tRNA reads. in the total RNA

522 sequencing (without polyA selection), and the scale factors for footprints were calculated from

523 the fraction of host reads from the total aligned ribosome profiling reads, including viral and host

524 reads.

525 Since the viral RNAs are widely overlapping, RNA-seq RPKM levels of viral genes were

526 computed with deconvolution as detailed here. First, values for each gene were calculated by

527 subtracting the RPKM of an ORF from the RPKM of the ORF located just upstream of it in the

528 genome. Then, for subgenomic RNAs, leader-body junction counts were counted based on

529 STAR alignment number of uniquely mapped reads crossing the junction. Finally, based on the

530 correlation between the deconvoluted RPKM and junction abundance of the subgenomic RNAs, 
531 the RPKM levels of all viral RNAs was estimated. To quantify the translation levels of non-

532 canonical viral ORFs ORF-RATER was used ${ }^{49}$.

\section{Quantification of intronic reads}

534 To avoid biases from intron read count, genes without introns, or where at least one of the

535 introns is overlapping with an exon of another gene were excluded. In addition, genes with low

536 number of reads ( $<20$ on the exons, $<2$ on the introns) were ignored. The number of reads on

537 exons and introns was normalized by the total length of the exons and introns respectively to get

538 quantification proportional to the number of molecules. Finally, the normalized number of reads

539 on the introns was calculated as percentage of the normalized number of reads on the exons.

540 Statistical significance (in figure 3E) was tested using a paired t-test on the log values of the

541 percentage (with offset of 0.1 to overcome zero values).

\section{Comparison of cytosolic lncRNAs and mitochondrial mRNAs}

543 The list of cytosolic lncRNAs is based on ${ }^{41}$. The list of $14 \operatorname{lncRNAs}$ (with number of reads $>=$

54450 ) that were used in the analysis are given in supplementary table 2 . The lncRNAs were

545 compared to protein coding genes, and mitochondrial encoded mRNAs were compared to

546 nuclear encoded mRNAs. Statistical significance was tested using Wilcoxon rank test on the log

547 fold change (compared to uninfected) of the RPKM values.

$548 \quad$ Protein synthesis measurement using O-Propargyl Puromycin (OPP)

549 OPP assay (OPP, Thermo Fisher Scientific) was carried out following the manufacturer's

550 instructions. Briefly, cells were collected following treatment with $10 \mu \mathrm{M}$ O-Propargyl

551 Puromycin for 30 minutes at $37^{\circ} \mathrm{C}$. The cells were then fixed for 15 minutes in $3.7 \%$

552 formaldehyde, and permeabilized in $0.1 \%$ Triton X-100 for 15 minutes. OPP was then

553 fluorescently labeled by a 30 minute incubation in Click-iT® Plus OPP reaction cocktail with

554 Alexa Fluor®594 picolyl azide (Thermo Fisher Scientific). Cells were analyzed using BD LSRII

555 flow cytometer. 
$\underline{\text { Pathway enrichment analysis }}$

558 Enrichment analysis of cellular pathways in specific gene clusters was done with PANTHER

559 version 15.0, with default settings and the PANTHER pathways data set ${ }^{54,55}$.

$\underline{\text { Reporter assay }}$

pLVX-EF1alpha-SARS-CoV-2-nsp1-2XStrep-IRES-Puro and pLVX-EF1alpha-SARS-CoV-2nsp2-2XStrep-IRES-Puro were kindly provided by Nevan Krogan, University of California, San

563 Francisco.

564 The viral 5'UTR was constructed based on nucleotides 4-265 of the reported sequence of SARS-

565 CoV-2 isolate Wuhan-Hu-1 (NC_045512.2) by sequential annealing of DNA oligonucleotides

566 (IDT, 5'UTR oligo_1-5 listed in the table below). The coding sequence for the first 12 amino

567 acids (aa) of ORF1a as well as the AcGFP homology region were added to the 5'UTR by two

568 PCR amplifications. For the 5'UTR-less control plasmid, the 12 aa region with AcGFP

569 homology was ordered from Sigma-Aldrich as oligonucleotides. The viral 5'UTR with the 12 aa

570 region and the 12 aa region on its own were cloned into pAcGFP1-C1 (Takara Biotech) using

571 restriction-free cloning. The entire expression cassette from the promoter to the poly-A site was

572 amplified and cloned into pDecko-mCherry (Addgene plasmid \#78534) using restriction-free

573 cloning. Primers for PCR amplification of fragments were ordered from Sigma-Aldrich. All

574 primers and oligonucleotides used for cloning are listed in the table below.

\begin{tabular}{|l|l|}
\hline Primer & Sequence \\
\hline 5'UTR oligo_1 & $\begin{array}{l}\text { caaGGTACCaaaggtttataccttccaggtaacaaaccaaccaactttcgatctct } \\
\text { tgtagatctgttctctaaacgaa }\end{array}$ \\
\hline 5'UTR oligo_2 & $\begin{array}{l}\text { Tgcgtgagtgcactaagcatgcagccgagtgacagccacacagattttaaagttcgttt } \\
\text { agagaacagatctacaagaga }\end{array}$ \\
\hline
\end{tabular}




\begin{tabular}{|c|c|}
\hline 5'UTR oligo_3 & $\begin{array}{l}\text { Catgcttagtgcactcacgcagtataattaataactaattactgtcgttgacaggacacg } \\
\text { agtaactcgtctatcttctg }\end{array}$ \\
\hline 5'UTR oligo_4 & $\begin{array}{l}\text { Cgaaacctagatgtgctgatgatcggctgcaacacggacgaaaccgtaagcagcctg } \\
\text { cagaagatagacgagttactcgt }\end{array}$ \\
\hline 5'UTR oligo_5 & atcatcagcacatctaggtttcgtccgggtgtgaccgaaaggtaagGGATCCaac \\
\hline $\begin{array}{l}\text { UTR_Amplification_round_1 } \\
\text { _Fwd }\end{array}$ & $\begin{array}{l}\text { GGTCTATATAAGCAGAGCTGGTTTAGTGAACCGattaaa } \\
\text { ggtttataccttcccaggtaacaaacc }\end{array}$ \\
\hline $\begin{array}{l}\text { UTR_Amplification_round_1 } \\
\text { _Rev }\end{array}$ & $\begin{array}{l}\text { GAAACCAGGGACAAGGCTCTCCATcttacctttcggtcacaccc } \\
\text { ggacg }\end{array}$ \\
\hline $\begin{array}{l}\text { UTR_Amplification_round_2 } \\
\text { _Fwd }\end{array}$ & GGTCTATATAAGCAGAGCTGGTTTAGTGAACCG \\
\hline $\begin{array}{l}\text { UTR_Amplification_round_2 } \\
\text { _Rev }\end{array}$ & $\begin{array}{l}\text { GAACAGCTCGGCGCCCTTGCTCACtgttttctcgttgaaaccagg } \\
\text { gacaaggctctcc }\end{array}$ \\
\hline 1-12 AA insert Fwd & $\begin{array}{l}\text { GGTCTATATAAGCAGAGCTGGTTTAGTGAACCGATgg } \\
\text { agagccttgtccetggtttcaacgagaaaacaGTGAGCAAGGGCGCCG } \\
\text { AGCTGTTCACC }\end{array}$ \\
\hline 1-12 AA insert Rev & $\begin{array}{l}\text { GGTGAACAGCTCGGCGCCCTTGCTCACtgttttctcgttgaaac } \\
\text { cagggacaaggctctccATCGGTTCACTAAACCAGCTCTGCTT } \\
\text { ATATAGACC }\end{array}$ \\
\hline pDecko cloning Fwd & $\begin{array}{l}\text { ccatttgtctcaagatctagttacgccaagcttcgttacataacttacggtaaatggcccg } \\
\text { cctggctg }\end{array}$ \\
\hline
\end{tabular}


293T cells were transfected using JetPEI (Polyplus-transfection) following the manufacturer's

578 instructions. Cells were assayed 24 hours post transfection by flow cytometry on a BD Accuri C6

579 flow cytometer and imaged on a Zeiss AxioObserver Z1 wide-field microscope using a X20

580 objective and Axiocam 506 mono camera.

583 Total RNA was extracted using Tri-Reagent (Sigma) and polyA selected using the Dynabeads

584 mRNA DIRECT purification kit (Thermo Fisher Scientific ) following the manufacturer's

585 instructions. cDNA was prepared using qScript cDNA Synthesis Kit (Quanta Biosciences)

586 following the manufacturer's instructions. Real time PCR was performed using the SYBR Green

587 PCR master-mix (ABI) on the QuantStudio 12K Flex (ABI) with the following primers (forward, 588 reverse):

589 ActinB (GTCATTCCAAATATGAGATGCGT, GCTATCACCTCCCCTGTGTG)

590 AcGFP (TGACCCTGAAGTTCATCTGC, GAAGTCGTGCTGCTTCATGT)

591 mCherry (ACCGCCAAGCTGAAGGTGAC, GACCTCAGCGTCGTAGTGGC)

592 GFP and mCherry mRNA levels were calculated relative to the human ActinB transcript. 


\section{References:}

596 1. Zhu, N. et al. A novel coronavirus from patients with pneumonia in China, 2019. N. Engl.

2. Zhou, P. et al. A pneumonia outbreak associated with a new coronavirus of probable bat origin. Nature 579, 270-273 (2020).

3. Sola, I., Almazán, F., Zúñiga, S. \& Enjuanes, L. Continuous and Discontinuous RNA Synthesis in Coronaviruses. Annu. Rev. Virol. 2, 265-288 (2015).

4. Stadler, K. et al. SARS — beginning to understand a new virus. Nat. Rev. Microbiol. 1, 209-218 (2003).

5. Snijder, E. J., Decroly, E. \& Ziebuhr, J. The Nonstructural Proteins Directing Coronavirus RNA Synthesis and Processing. in Advances in Virus Research vol. 96 59-126 (Academic

6. V'kovski, P., Kratzel, A., Steiner, S., Stalder, H. \& Thiel, V. Coronavirus biology and replication: implications for SARS-CoV-2. Nat. Rev. Microbiol. 1-16 (2020) doi:10.1038/s41579-020-00468-6. Press Inc., 2016).

8. Davidson, A. D. et al. Characterisation of the transcriptome and proteome of SARS-CoV2 reveals a cell passage induced in-frame deletion of the furin-like cleavage site from the spike glycoprotein. Genome Med. 12, 68 (2020). doi:10.1038/s41586-020-2739-1.

10. Stern-Ginossar, N., Thompson, S. R., Mathews, M. B. \& Mohr, I. Translational control in virus-infected cells. Cold Spring Harb. Perspect. Biol. 11, a033001 (2019). 
619 11. Streicher, F. \& Jouvenet, N. Stimulation of Innate Immunity by Host and Viral RNAs.

620 Trends in Immunology vol. 40 1134-1148 (2019).

621 12. Schoggins, J. W. Interferon-Stimulated Genes: What Do They All Do? Annu. Rev. Virol. $622 \quad 6,567-584(2019)$.

623 13. Ribero, M. S., Jouvenet, N., Dreux, M. \& Nisole, S. Interplay between SARS-CoV-2 and 624 the type I interferon response. PLoS Pathogens vol. 16 e1008737 (2020).

625 14. Meffre, E. \& Iwasaki, A. Interferon deficiency can lead to severe COVID. Nature (2020) 626 doi:10.1038/d41586-020-03070-1.

627 15. Hadjadj, J. et al. Impaired type I interferon activity and inflammatory responses in severe 628 COVID-19 patients. Science (80-. ). 369, 718-724 (2020).

629 16. Bastard, P. et al. Autoantibodies against type I IFNs in patients with life-threatening 630 COVID-19. Science 370, (2020).

631 17. Zhang, Q. et al. Inborn errors of type I IFN immunity in patients with life-threatening COVID-19. Science 370, (2020).

633 18. Blanco-Melo, D. et al. Imbalanced Host Response to SARS-CoV-2 Drives Development of COVID-19. Cell 181, 1036 (2020).

19. Wyler, E. et al. Bulk and single-cell gene expression profiling of SARS-CoV-2 infected human cell lines identifies molecular targets for therapeutic intervention. (2020) doi:10.1101/2020.05.05.079194.

20. Abernathy, E. \& Glaunsinger, B. Emerging roles for RNA degradation in viral replication and antiviral defense. Virology 479-480, 600-608 (2015).

640 21. Kamitani, W., Huang, C., Narayanan, K., Lokugamage, K. G. \& Makino, S. A twopronged strategy to suppress host protein synthesis by SARS coronavirus Nsp1 protein. Nat. Struct. Mol. Biol. 16, 1134-1140 (2009). 
643 22. Lokugamage, K. G. et al. Middle East Respiratory Syndrome Coronavirus nsp1 Inhibits

644 Host Gene Expression by Selectively Targeting mRNAs Transcribed in the Nucleus while Sparing mRNAs of Cytoplasmic Origin. J. Virol. 89, 10970-10981 (2015).

646 23. Nakagawa, K., Lokugamage, K. G. \& Makino, S. Viral and Cellular mRNA Translation in Coronavirus-Infected Cells. in Advances in Virus Research vol. 96 165-192 (Academic Press Inc., 2016).

24. Banerjee, A. K. et al. SARS-CoV-2 Disrupts Splicing, Translation, and Protein Trafficking to Suppress Host Defenses. Cell 183, 1-15 (2020).

25. Thoms, M. et al. Structural basis for translational shutdown and immune evasion by the Nsp1 protein of SARS-CoV-2. Science vol. 369 http://science.sciencemag.org/ (2020).

26. Lapointe, C. P. et al. Dynamic competition between SARS-CoV-2 NSP1 and mRNA on the human ribosome inhibits translation initiation. bioRxiv 2020.08.20.259770 (2020) doi:10.1101/2020.08.20.259770.

27. Schubert, K. et al. SARS-CoV-2 Nsp1 binds the ribosomal mRNA channel to inhibit translation. Nat. Struct. Mol. Biol. 27, 959-966 (2020).

28. Shi, M. et al. SARS-CoV-2 Nsp1 suppresses host but not viral translation through a bipartite mechanism. doi:10.1101/2020.09.18.302901.

29. Addetia, A. et al. SARS-CoV-2 ORF6 disrupts nucleocytoplasmic transport through interactions with Running title: SARS-CoV-2 ORF6 disrupts mRNA nuclear export. bioRxiv 2020.08.03.234559 (2020) doi:10.1101/2020.08.03.234559.

30. Xia, H. et al. Evasion of Type I Interferon by SARS-CoV-2. Cell Rep. 33, 108234 (2020). stem cells reveals the complexity and dynamics of mammalian proteomes. Cell 147, 789802 (2011). 
667 32. Stern-Ginossar, N. \& Ingolia, N. T. Ribosome Profiling as a Tool to Decipher Viral

668 Complexity. Annu. Rev. Virol. 2, 335-349 (2015).

669 33. Wolff, G. et al. A molecular pore spans the double membrane of the coronavirus

670 replication organelle. Science (80-. ). 369, 1395-1398 (2020).

671 34. Liu, J., Xu, Y., Stoleru, D. \& Salic, A. Imaging protein synthesis in cells and tissues with 672 an alkyne analog of puromycin. Proc. Natl. Acad. Sci. U. S. A. 109, 413-418 (2012).

673 35. Del Valle, D. M. et al. An inflammatory cytokine signature predicts COVID-19 severity 674 and survival. Nat. Med. 26, 1636-1643 (2020).

675 36. Irigoyen, N. et al. High-Resolution Analysis of Coronavirus Gene Expression by RNA 676 Sequencing and Ribosome Profiling. PLoS Pathog. 12, 1005473 (2016).

677 37. Firth, A. A putative new SARS-CoV protein, 3c, encoded in an ORF overlapping ORF3a. J. Gen. Virol. jgv001469 (2020) doi:10.1099/jgv.0.001469.

679 38. Jungreis, I., Sealfon, R. \& Kellis, M. Sarbecovirus comparative genomics elucidates gene content of SARS-CoV-2 and functional impact of COVID-19 pandemic mutations. bioRxiv 2020.06.02.130955 (2020) doi:10.1101/2020.06.02.130955.

39. Cagliani, R., Forni, D., Clerici, M. \& Sironi, M. Coding potential and sequence conservation of SARS-CoV-2 and related animal viruses. Infect. Genet. Evol. 83, (2020).

40. Hinnebusch, A. G. Molecular Mechanism of Scanning and Start Codon Selection in Eukaryotes. Microbiol. Mol. Biol. Rev. 75, 434-467 (2011).

41. Zuckerman, B., Ron, M., Mikl, M., Segal, E. \& Ulitsky, I. Gene Architecture and Sequence Composition Underpin Selective Dependency of Nuclear Export of Long RNAs on NXF1 and the TREX Complex. Mol. Cell 79, 251-267.e6 (2020). 
43. Tidu, A. et al. The viral protein NSP1 acts as a ribosome gatekeeper for shutting down host translation and fostering SARS-CoV-2 translation Runing title: SL1 controls NSP1 for SARS-CoV-2 viral translation. bioRxiv 2020.10.14.339515 (2020)

44. Kim, D. et al. The architecture of SARS-CoV-2 transcriptome. Cell S0092-8674, 30406-2 (2020).

45. Thiel, V. et al. Mechanisms and enzymes involved in SARS coronavirus genome expression. Journal of General Virology vol. 84 2305-2315 (2003).

46. Huang, C. et al. Alphacoronavirus Transmissible Gastroenteritis Virus nsp1 Protein Suppresses Protein Translation in Mammalian Cells and in Cell-Free HeLa Cell Extracts

47. Huang, C. et al. SARS coronavirus nsp1 protein induces template-dependent endonucleolytic cleavage of mRNAs: Viral mRNAs are resistant to nsp1-induced RNA cleavage. PLoS Pathog. 7, e1002433 (2011).

48. Gordon, D. E. et al. A SARS-CoV-2 protein interaction map reveals targets for drug repurposing. Nature 583, 459-468 (2020).

49. Fields, A. P. et al. A Regression-Based Analysis of Ribosome-Profiling Data Reveals a Conserved Complexity to Mammalian Translation. Mol. Cell 60, 816-827 (2015).

50. Finkel, Y. et al. Comprehensive annotations of human herpesvirus 6A and 6B genomes reveal novel and conserved genomic features. Elife 9, e50960 (2020).

712 51. Tirosh, O. et al. The Transcription and Translation Landscapes during Human 713 Cytomegalovirus Infection Reveal Novel Host-Pathogen Interactions. PLoS Pathog. 11, e1005288 (2015). 
715 52. Langmead, B., Trapnell, C., Pop, M. \& Salzberg, S. L. Ultrafast and memory-efficient

716 alignment of short DNA sequences to the human genome. Genome Biol. 10, R25 (2009).

717 53. Dobin, A. et al. STAR: ultrafast universal RNA-seq aligner. Bioinformatics 29, 15-21

718 (2013).

719 54. Mi, H. \& Thomas, P. PANTHER pathway: an ontology-based pathway database coupled 720 with data analysis tools. Methods Mol. Biol. 563, 123-140 (2009).

721 55. Mi, H., Muruganujan, A., Ebert, D., Huang, X. \& Thomas, P. D. PANTHER version 14:

722 more genomes, a new PANTHER GO-slim and improvements in enrichment analysis

723 tools. Nucleic Acids Res. 47, 419-426 (2018). 
bioRxiv preprint doi: https://doi.org/10.1101/2020.11.25.398578; this version posted November 25, 2020. The copyright holder for this preprint (which was not certified by peer review) is the author/funder, who has granted bioRxiv a license to display the preprint in perpetuity. It is made available under aCC-BY-NC 4.0 International license.

a

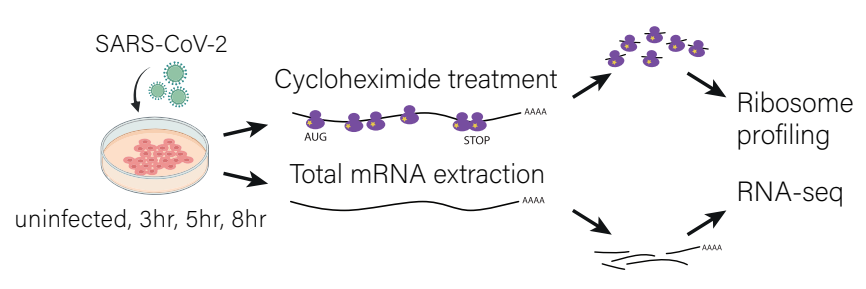

b

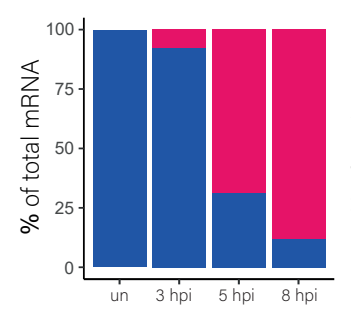

C

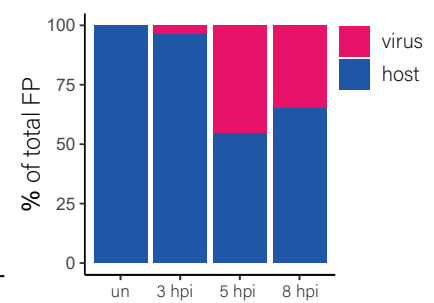

d

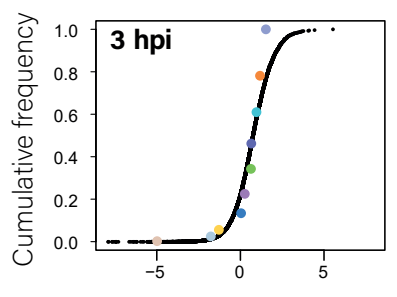

e

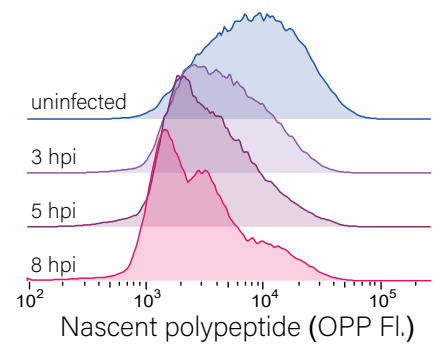

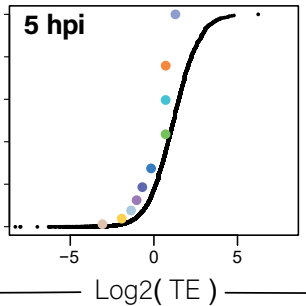

$\mathbf{f}$

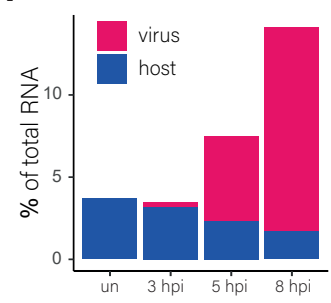

h

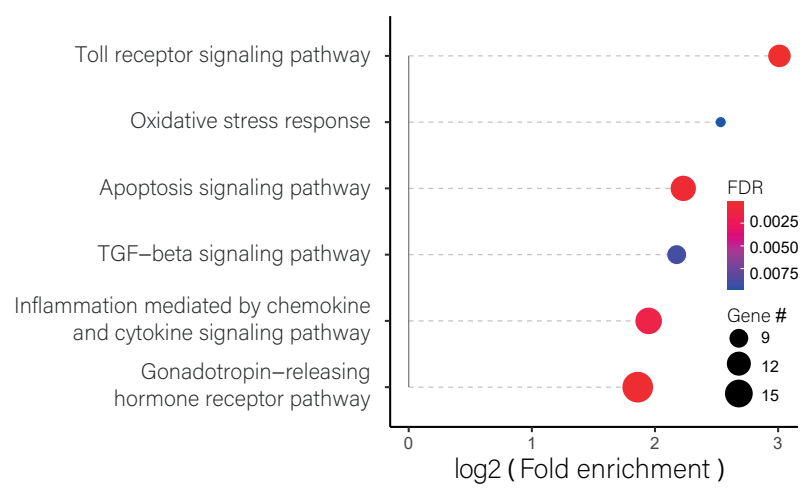

g
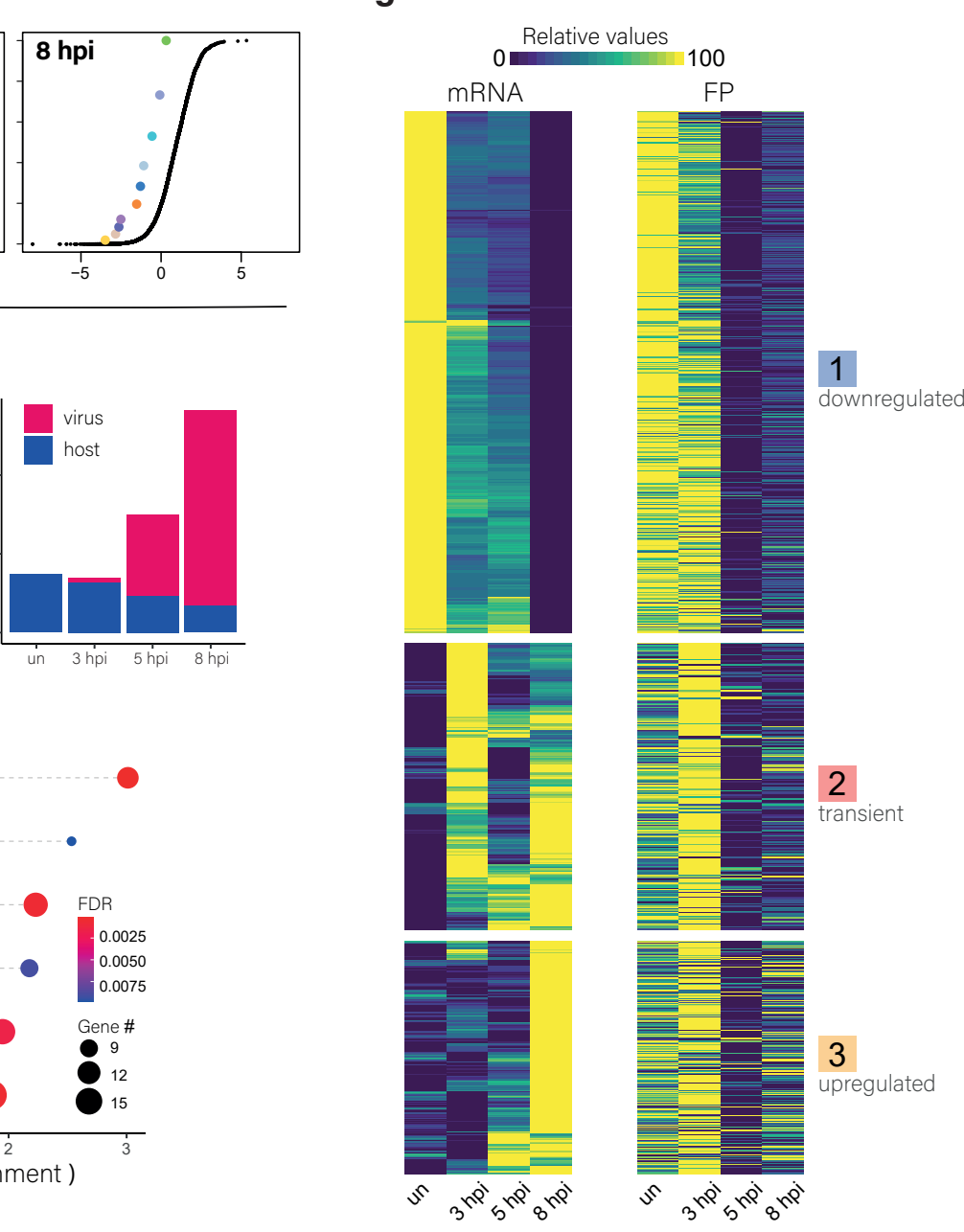

Figure 1: Global reduction of translation and in cellular mRNA levels along SARS-CoV-2 Infection

(A) Calu3 cells were left uninfected or were infected with SARS-CoV-2 (MOI=3) for 3, 5 or 8 hours and harvested for RNA-seq and for Ribo-seq. (B and C) Percentage of reads that aligned to human or viral transcripts from the sum of aligned reads shown for mRNAs (B) and footprints (C) in uninfected cells and in cells harvested at 3, 5 and 8 hpi. (D) Cumulative TE distribution among wellexpressed human (black points) and viral (colored points) genes at 3, 5 and 8 hpi. (E) Protein synthesis measurement by flow cytometry of Calu3 cells infected with SARS-CoV-2 (MOI = 3) for 3, 5 and 8 hpi or an uninfected control following O-Propargyl Puromycin (OPP) incorporation and fluorescent labelling. (F) Percent of reads that aligned to the human or viral transcripts from the sum of total RNA reads in uninfected cells and in cells harvested at, 3, 5 and 8 hpi. (G) Heat map presenting relative mRNA and footprints expression of well-expressed human transcripts that showed the most significant changes in their mRNA levels at 8 hpi relative to uninfected, across time points during SARS-CoV-2 infection. Shown are expression levels scaled by gene after partitioning clustering. Three main clusters are marked on the right. (H) Summary of pathway enrichment analysis of genes enriched in cluster 3 (upregulated genes). Dot size reflects the number of genes from each pathway included in the tested set, and dot color reflects the false discovery rate (FDR) of the pathway enrichment. 
bioRxiv preprint doi: https://doi.org/10.1101/2020.11.25.398578; this version posted November 25, 2020. The copyright holder for this preprint (which was not certified by peer review) is the author/funder, who has granted bioRxiv a fieense to display the preprint in perpetuity. It is made available under aCC-BY-NC 4.0 International license.

b

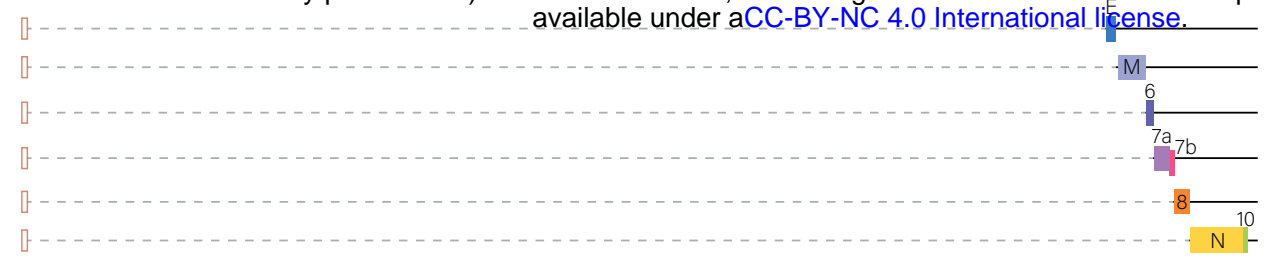

d

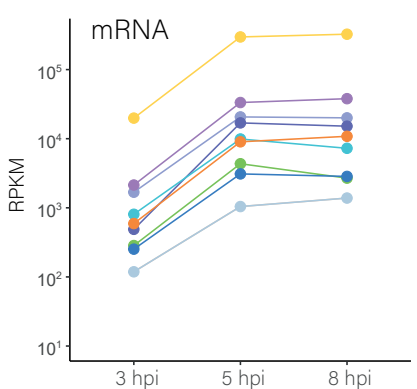

C
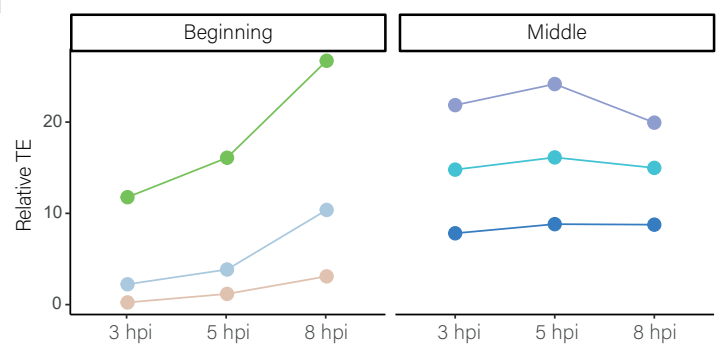

10000

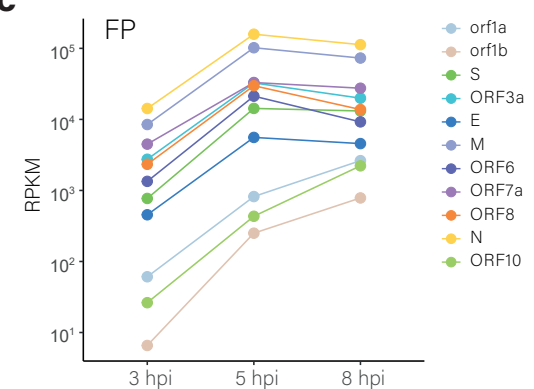

e

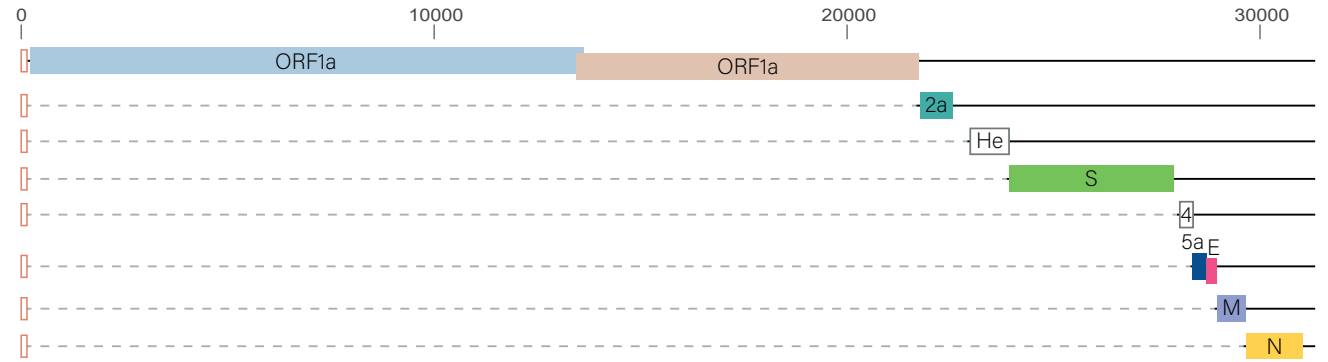

f

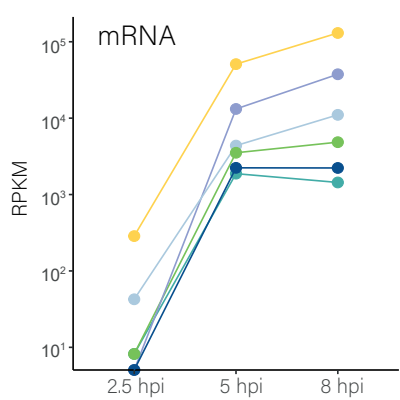

g

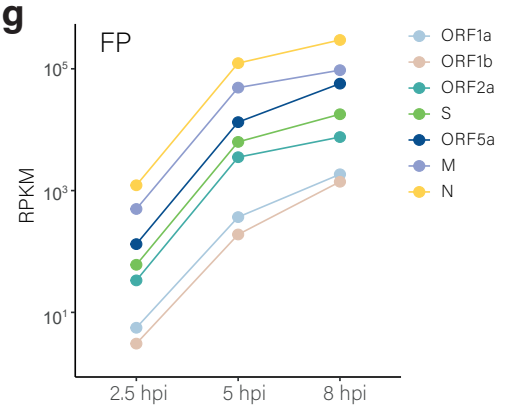

h

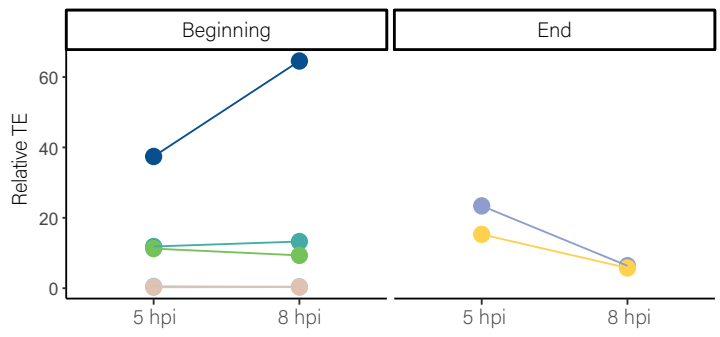

i
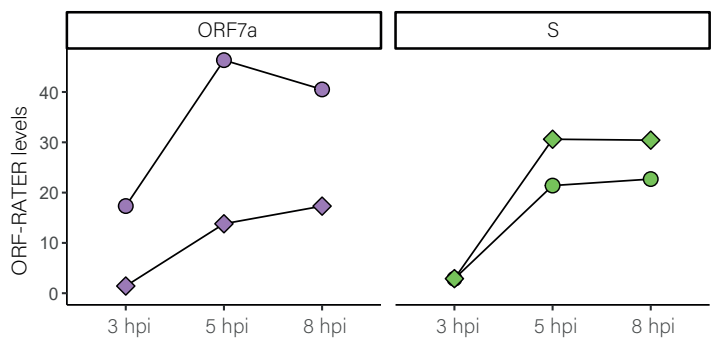

- orf1a

-0 orf1b

- ORF3a

$\rightarrow-E$

- M ORF6

- ORF7a

- ORF8

$\rightarrow$ ORF10 and MHV infection densities using ORF-RATER ${ }^{49}$.

Figure 2: Changes in viral gene expression along SARS-CoV-2

(A) Schematic presentation of the SARS-CoV-2 genome organization, the subgenomic mRNAs and the main ORFs. (B and C) RNA (B) and translation levels (C) of each of the SARS-CoV2 canonical ORFs at 3, 5 and 8 hpi. (D) Relative translation efficiency of each canonical viral ORF along infection. Genes are divided to three groups based on their physical location along the genome. (E) Schematic presentation of the MHV subgenomic mRNAs, and the main ORFs. ORFs that are not expressed in the strain that was used for ribosome profiling (MHV-A59) are marked by empty rectangles. (F and G) RNA (F) and translation levels (G) of each of the MHV ORFs at 2.5, 5 and 8 hpi.

Expression was calculated from ${ }^{36}$. (H) Relative translation efficiency of each canonical viral ORF along infection. Genes are divided to two groups based on their location on the genome. (I) Relative translation efficiency of the main ORFs (ORF7a, S and ORF3a, labeled by circle) and the out of frame ORFs (ORF7b, S.iORF and ORF3c, labeled by diamond) of the S, ORF3a and ORF7a subgenomic transcripts, respectively, along SARS-CoV-2 infection. Translation levels were calculated from ribosome 
$p<10^{-16}$

$\mathrm{p}<10^{-16}$ $p<10^{-16}$

abioRxiv preprint doi: https://doi.org/10-1101/2020.11.25.398578; this version posted November 25, 2020. The copyright holder for this preprint (whigh 3/kas not certified by peer revievs)hiss the author/funder, who hassgifanted bioRxiv a license to display the preprint in perpetuity. It is made E available under aCG-BY-NC 4.0 International license.
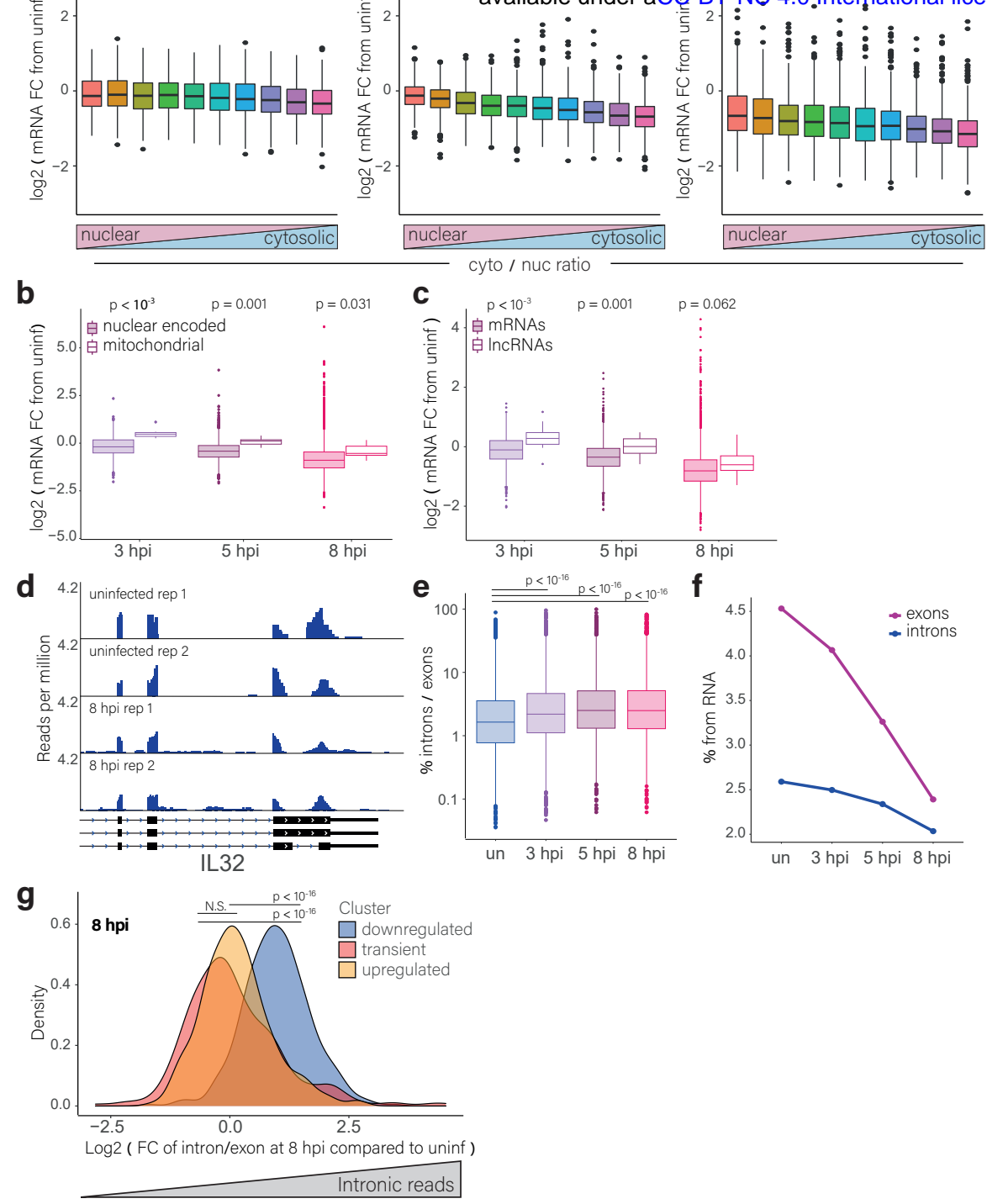

Figure 3: Cytosolic cellular RNAs are degraded during SARS-CoV-2 infection

(A) RNA level fold change of cellular RNAs at different time points after infection relative to uninfected cells. RNAs were grouped to ten bins based on their cytosol to nucleus localization ratio ${ }^{41}$.

(B) The change in RNA levels of nuclear encoded or mitochondrial encoded RNAs at different time points after infection relative to uninfected cells. (C) The change in RNA expression of cytosolic lncRNAs and protein coding mRNAs at different time points after infection relative to uninfected cells. (D) RNA reads on exons and introns of the end of IL-32 gene from uninfected cells and at 8 hpi. (E) Box plots presenting the ratio of intronic to exonic reads for each gene in uninfected cells and at the different time points along SARSCoV-2 infection. (F) The \% of reads that align to exonic or intronic regions relative to rRNA abundance along SARS-CoV-2 infection. (H) Histograms showing the change in the ratio of intronic to exonic reads of cellular genes at $8 \mathrm{hpi}$ relative to uninfected cells. Genes are divided according to the three clusters shown in figure $1 \mathrm{G}$ (representing different expression pattern along infection). 
Figure 4

bioRxiv preprint doi: https://doi.org/10.1101/2020.11.25.398578; this version posted November 25, 2020. The copyright holder for this preprint (ahich was not certified by peer review) is the author/funder, who has granted bioRxiv a license to display the preprint in perpetuity. It is made

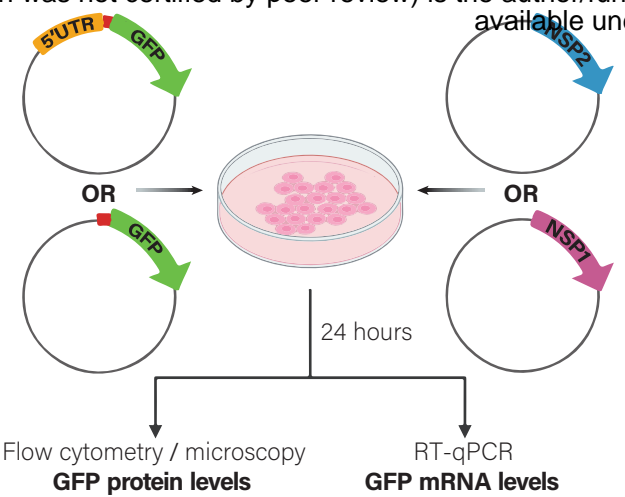

b

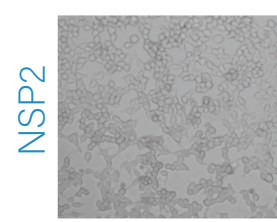

GFP (-) 5'UTR
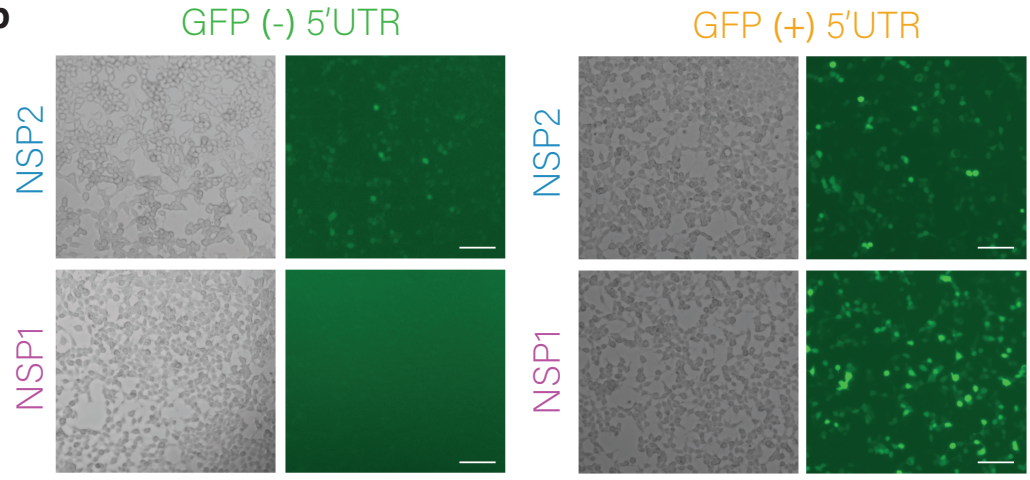

C
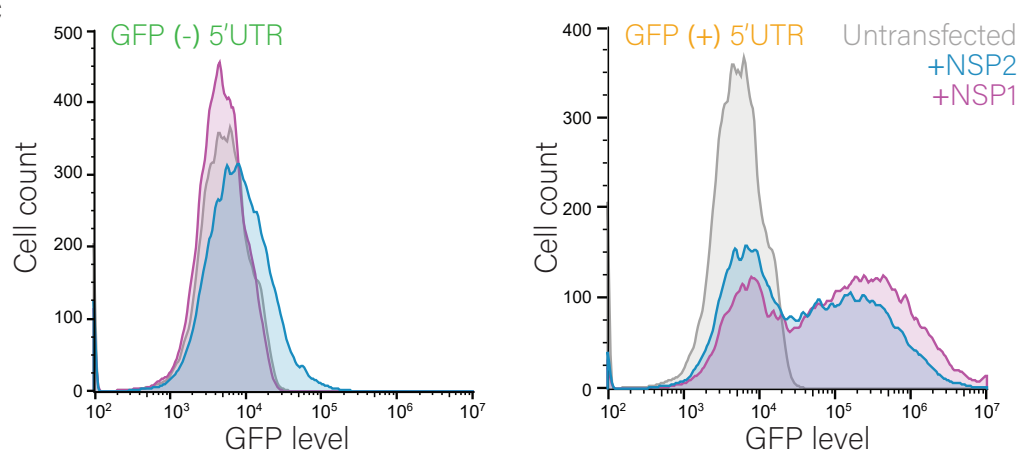

d
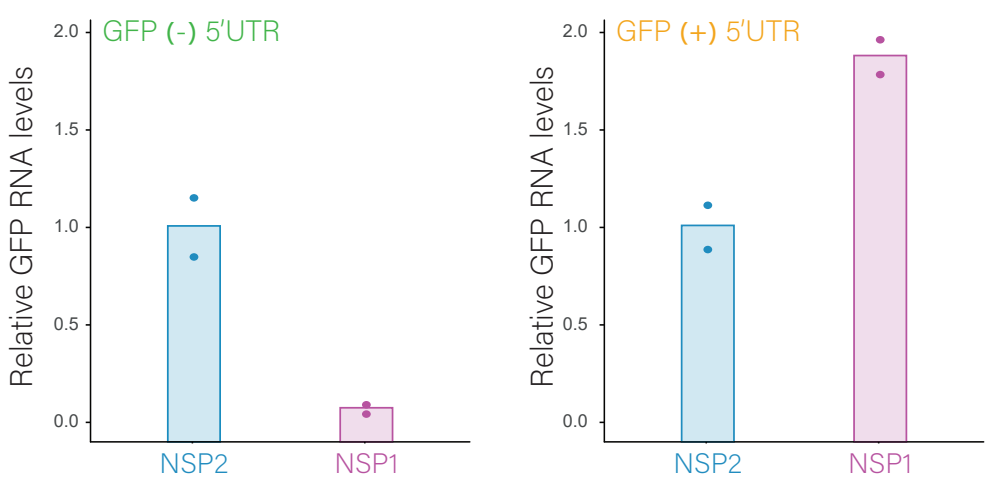

Figure 4: The viral 5' UTR protects mRNA from NSP1-mediated degradation

(A) $293 \mathrm{~T}$ cells were transfected with expression vectors encoding either NSP1 or NSP2 (as a control) and with a GFP reporter (GFP (-) 5'UTR) or a GFP reporter that includes the viral 5'UTR (GFP (+) 5'UTR). (B)

Microscopy images of cells co-transfected with NSP2 (top) or NSP1 (bottom) together with either GFP (-) 5'UTR or GFP (+) 5'UTR. Scale bars are $100 \mu \mathrm{m}$. (C) Flow cytometry analysis of cells co-transfected with NSP1 or NSP2 together with either GFP (-) 5'UTR or GFP (+) 5'UTR. (D) Relative GFP RNA levels from GFP (-) 5'UTR or GFP (+) 5'UTR in cells expressing NSP1 or NSP2 as measured by quantitative RT-PCR. Data points show measurement of biological replicates. Shown is one representative experiment out of two performed. 


\section{Figure 5}

bioRxiv preprint doi: https://doi.org/10.1101/2020.11.25.398578; this version posted November 25, 2020. The copyright holder for this preprint (which was not certified by peer review) is the author/funder, who has granted bioRxiv a license to display the preprint in perpetuity. It is made

a
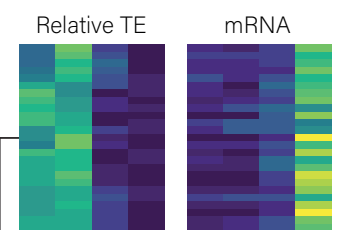

FP available unqfor
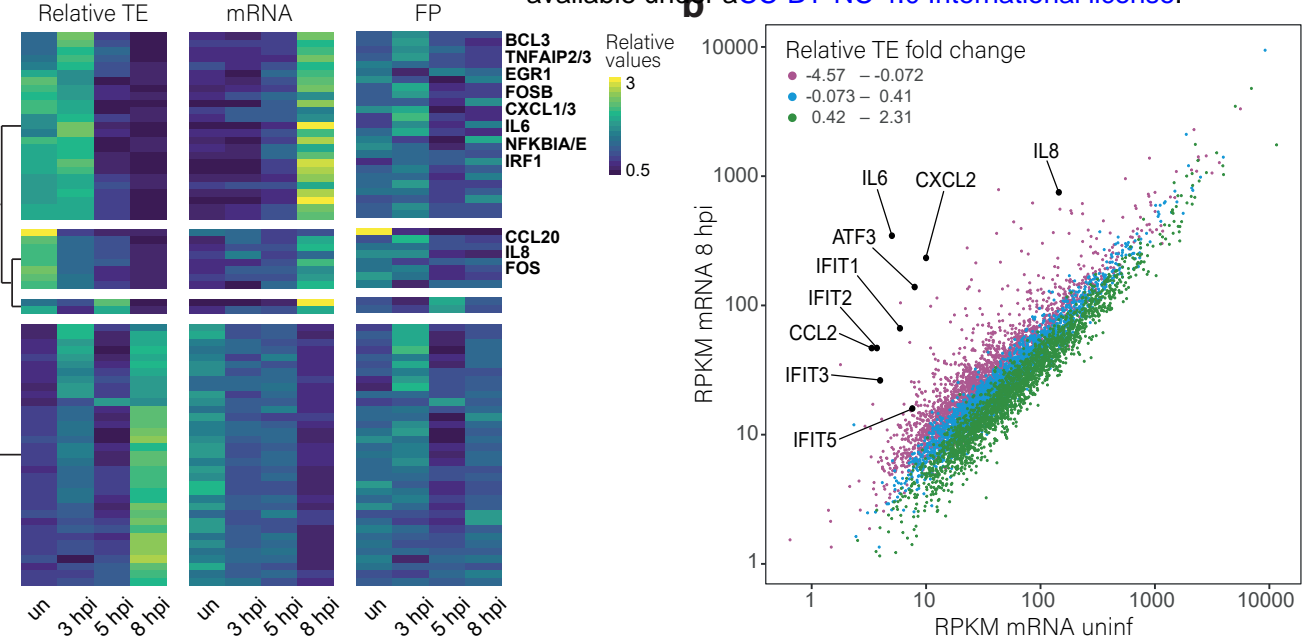

C
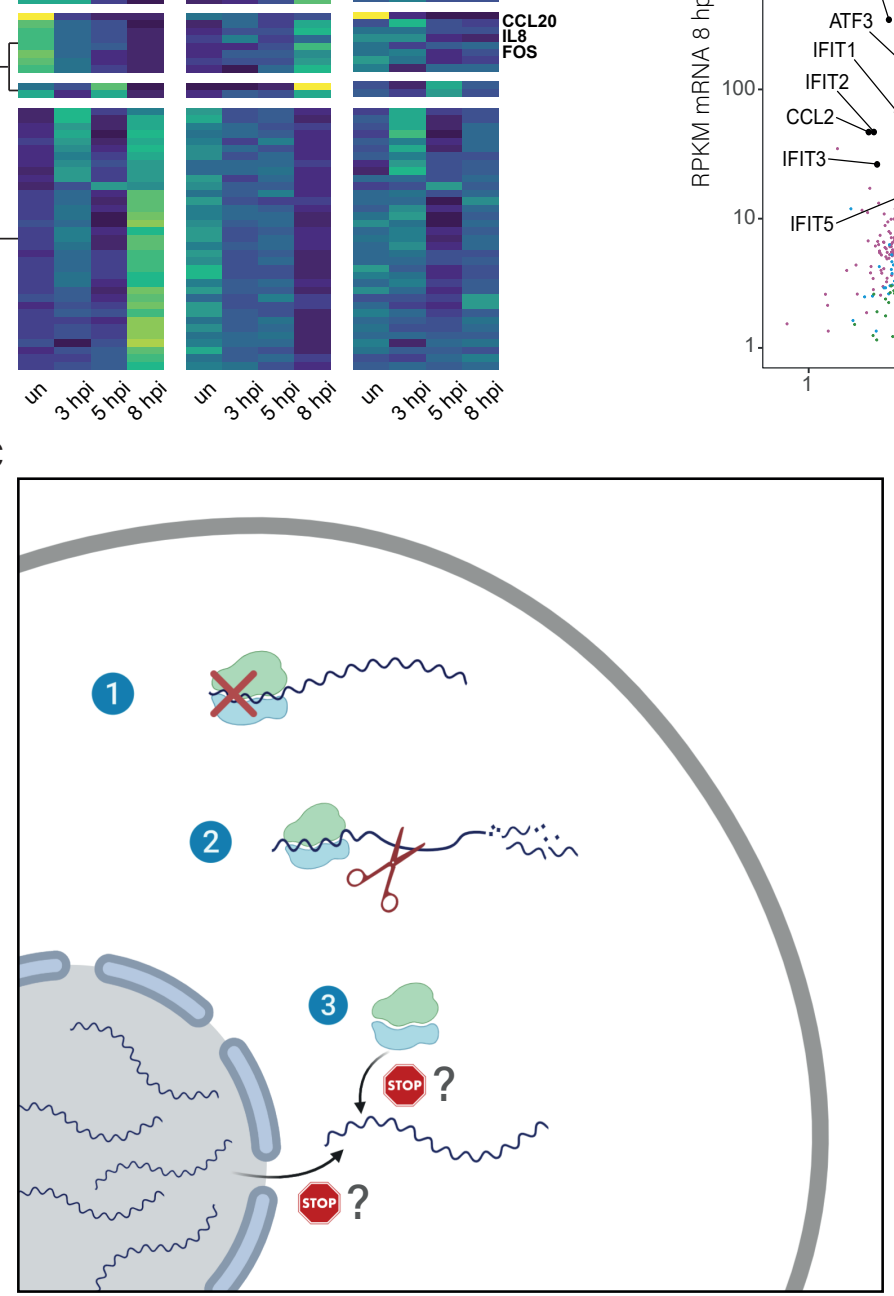

Figure 5: The translation of induced transcripts is impaired during infection

(A) Heat map presenting relative TE, mRNA and footprints (FP) of human genes that showed the most significant changes in their relative TE along SARS-CoV-2 infection. Shown are relative expression ratios after partitioning clustering based on changes in relative TE values. (B) Scatter plot presenting cellular transcript levels in uninfected cells compared to 8hpi. Genes are colored based on the relative change in their TE between uninfected and 8hpi. Central cytokines and IFN stimulated genes are labeled. (C) A model of how SARS-CoV-2 suppresses host gene expression through multi-pronged approach: 1. Global translation reduction; 2. Degradation of cytosolic cellular mRNAs; 3 . Specific translation inhibition of newly synthesized cellular mRNAs. 